\title{
Happy Hour? A Preliminary Study of the Effect of Induced Joviality and Sadness on Beer Perception
}

\author{
Beth Desira ${ }^{1}$, Shaun Watson ${ }^{1}\left(\mathbb{D}\right.$, George Van Doorn ${ }^{2, *}$, Justin Timora ${ }^{3}\left(\mathbb{D}\right.$ and Charles Spence ${ }^{4}(\mathbb{C})$ \\ 1 School of Health and Life Sciences, Mt Helen Campus, Federation University Australia, \\ Ballarat, VIC 3350, Australia; bethldesira@students.federation.edu.au (B.D.); \\ shaun.watson@federation.edu.au (S.W.) \\ 2 School of Health and Life Sciences, Churchill Campus, Federation University Australia, \\ Churchill, VIC 3842, Australia \\ 3 School of Health and Life Sciences, Berwick Campus, Federation University Australia, \\ Berwick, VIC 3806, Australia; j.timora@federation.edu.au \\ 4 Crossmodal Research Laboratory, Oxford University, Oxford OX2 6GG, UK; charles.spence@psy.ox.ac.uk \\ * Correspondence: george.vandoorn@federation.edu.au; Tel.: +61-3-5122-6746
}

Received: 6 April 2020; Accepted: 26 May 2020; Published: 1 June 2020

check for updates

\begin{abstract}
Our emotions influence our perception. In order to determine whether emotion influences the perception of beer, 32 participants watched either a scene from the movie Wall-E to induce joviality, or a short clip from the Shawshank Redemption to induce sadness. The participants were then required to sample up to $250 \mathrm{~mL}$ of Yenda Pale Ale beer and rate it on a variety of taste and flavor characteristics (e.g., bitterness), before completing the Positive and Negative Affect Schedule-X (PANAS-X). The data were analyzed using Bayesian $t$-tests and Null Hypothesis Significance Tests (NHSTs). After applying conservative corrections for multiple comparisons, NHSTs failed to reach statistical significance. However, the effect sizes suggested that inducing joviality, relative to inducing sadness, resulted in the beer being rated as (a) tasting more pleasant, (b) tasting sweeter, and (c) being of higher quality. Following the induction of joviality, participants were also willing to pay more for the beer. The Bayesian analyses indicated that induced emotion can influence flavor perception for complex taste stimuli. The effect sizes and Bayesian analyses are interpreted in terms of Feelings-as-Information theory. These preliminary findings can tentatively be applied to real-world environments such as venues that serve and/or market alcohol.
\end{abstract}

Keywords: Induced emotion; beer; flavor

\section{Introduction}

An extensive body of empirical research shows that our emotions influence our perception (see [1] for a review). For example, individuals experiencing happiness, relative to sadness, perceive hills to be less steep [2], and chocolate as tasting more pleasant [3]. At the outset, it is important to note that although the terms 'emotion' and 'mood' have historically been used interchangeably, they are, in fact, distinct, albeit related, constructs. Emotions typically have an identifiable referent (i.e., a specific source), a short onset time, and a limited duration. In contrast, moods may lack a clear referent, arise more gradually, are more diffuse, and tend to be of lower intensity. What is more, they may last for an extended period of time (see [4-7] for discussions of these differences). Here, we focus on emotions because, as noted by Côtè [8], the interpretation of the broad valence of mood (e.g., positive/negative, pleasant/unpleasant) is limited, while an examination of discrete emotional states may provide insight into specific patterns of appraisal.

Beer has a complex flavor profile $[9,10]$, which makes it a good candidate for research because its attributes are difficult to describe. Beer is also important from a social perspective, being one 
of the world's oldest manufactured drinks [11], not to mention one of the world's most consumed beverages [12]. In fact, beer is the single most consumed alcoholic beverage worldwide by volume [13]. In 2017, global beer consumption was approximately 186.82 million kiloliters [14]. Given how much beer is consumed annually, it is surprising that more research has not been published assessing the role played by emotions in the perceived taste of beer. This preliminary study is necessary and novel in that, although there is an extensive body of research examining the influence of emotions on taste/flavor perception, only one recent study has examined the influence of emotions, as induced by music, in the perception of beer (i.e., [15]). Our study differs from Reinoso-Carvalho et al.'s [15] prior work in this area in the methodology used, including the stimuli used and the emotions induced, the theoretical framework applied, the types of analyses conducted (i.e., Bayesian statistics), and the conclusions drawn.

\subsection{The Influence of Emotion on Taste/Flavor Perception}

An early study in this field investigated the influence of emotions on women's perception of cheese [16]. The participants were induced to feel either happiness or sadness by reading either positive or negative statements referring to the self. They then consumed a small amount of cheese and rated its hedonic qualities. Women induced to feel happiness rated the cheese as being more pleasant than those induced to feel sadness. In another study, Macht et al. [3] induced anger, fear, sadness, or joy via film clips, and asked healthy men to consume and rate a piece of chocolate. Of relevance, Macht et al. [3] found that men experimentally induced to feel joy rated the chocolate as tasting more pleasant than those induced to feel sadness.

Noel and Dando [17], meanwhile, had the spectators at hockey games taste and evaluate salted caramel pretzel ice-cream and lemon-lime sorbet. The researchers argued that the wins or losses of the participants' hockey teams would result in them feeling positive or negative emotions, respectively. Noel and Dando [17] found that when their team won, the participants reported sweet tastes as being more intense (i.e., sweeter), while sour tastes were rated as being less intense (i.e., as less sour). This was true regardless of the ice-cream/sorbet flavor under consideration. The authors also found that wins resulted in increased pleasantness ratings for the lemon-lime flavored sorbet. These findings are interesting but should be interpreted with caution given that emotion was not measured directly. Instead, winning was equated to positive affect, while losing was equated to negative affect. The authors conducted an 'affect validation study' using a card game to examine if wins and losses were indeed associated with positive and negative affect. Although Noel and Dando [17] found that the "win or loss of the card game successfully manipulated positive affect scores" (p. 91), they acknowledge the limitation of comparing personal success in an interactive card game to the supporters' experience at a sporting event. In a further possible confound, the winner in the card game received more money than did the loser. Thus, it might not be 'winning' that had a positive effect on emotion but, instead, the amount of money the person won (see [18] for an example of how monetary value affects taste perception).

Wang and Spence [19] examined whether the sensory and hedonic aspects of taste/flavor perception were influenced by auditory and visual stimuli. Specifically, the participants in their study were asked to taste and evaluate the sweetness, sourness, and hedonic properties of fruit juice while being exposed to (a) a harmonious melody (positive valence), (b) a dissonant melody (negative valence), (c) a static image of a child laughing, or (d) a static image of a child crying. All of the participants took part in each of the four conditions. When exposed to the pleasant stimuli (i.e., harmonious melody or an image of the laughing child), the participants rated the juice as tasting sweeter than when they were exposed to the unpleasant stimuli (i.e., dissonant melody or an image of the crying child) instead. The authors suggested that the emotional valence of food-extrinsic stimuli (e.g., the intrinsic pleasantness of the harmonious melody) influenced perception. This is a reasonable interpretation. However, it is possible that the mechanism by which this effect may have occurred is that the stimuli induced certain emotions 
(e.g., joy) which, in turn, influenced participants' perception. After all, it has been well-established that music influences emotions [20].

Lastly, Reinoso-Carvalho et al. [15] conducted a series of studies designed to determine if emotions, induced by music, would influence people's perception of beer. Specifically, participants were asked to taste and evaluate a beer while being exposed to positive or negative music. All of the participants completed both conditions. The results revealed that when exposed to positive music, people rated the beer as more likeable, as tasting sweeter, and were willing to pay approximately $8 \%$ more for it. The same beer was rated as (a) having a higher alcohol content, (b) being more bitter, and (c) having more body when consumed in the presence of music that was associated with negative emotions.

\subsection{Theoretical Framework (Feelings-as-Information Theory)}

Feelings-as-Information (FaI) theory $[7,21]$ may offer a theoretical explanation for the potential influence of emotions on perception. According to this theory, people use their current 'feelings' (as a synonym for mood and emotion) as a conscious or unconscious source of information in judgement [7]. Regardless of whether the target of judgement elicits these feelings or not, the feelings provide immediate information with which to evaluate an object, situation, or person. For example, a feeling of happiness may be used to infer that the current situation is good. In support of such a suggestion, Brackett, Floman, Ashton-James, Cherkasskiy, and Salovey [22] investigated evaluative judgements and found that teachers gave higher grades to students when induced to experience positive emotion, and lower grades when induced to experience negative emotion instead. In the context of the current research, a pleasant or positive emotion may therefore result in more pleasant or positive evaluations of beer.

In addition to feelings serving as a source of information that acts as a basis for judgment, it has been suggested that feelings also influence how individuals process information [7]. FaI theory postulates that negative/unpleasant feelings promote analytic, bottom-up processing, while positive/pleasant feelings promote global, top-down processing $[7,8]$. Experiencing negative feelings signals something problematic about one's environment, and trying to determine what is wrong and what can be done about it requires a more analytic evaluation of the current situation [7]. Thus, individuals experiencing negative feelings are more likely to focus on, and pay greater attention to, the details of their environment, relative to those experiencing positive feelings $[7,8]$. Opting for attention to detail, they are also less likely to rely on schemas and heuristics (e.g., mental short-cuts or rules-of-thumb that allow people to make decisions quickly) [23]. In contrast, $\mathrm{FaI}$ argues that positive feelings indicate that the current situation is benign $[7,8]$. As such, individuals have less motivation to alter their current state, and therefore have less need to conduct a thorough examination of their current situation. Instead, there is an increased tendency to perceive the situation more holistically [23]. Subsequently, these individuals are more likely to use heuristics, exert less effort, and opt for more global processing [7,24]. In the context of beer perception, this suggests that those in a negative state will be more analytic, while those in a positive state will conduct a more holistic assessment. An analytic approach to beer would result in greater scrutiny of the specific attributes of the beer (e.g., bitterness), with the person attempting to identify factors that are contributing to their current state.

\subsection{Aims and Hypotheses}

The present study is a preliminary examination of emotion and its influence on beer. To the best of the authors' knowledge, this is only the second study to do so. It used four descriptors (i.e., sweetness, bitterness, fruitiness, and intensity of flavor to assess the taste/flavor characteristics of beer. An 'electronic tongue' comprised of potentiometric chemical sensors identified several sensory attributes of beer including sour, sweet, bitter, fruity, intensity, caramel, artificial, burned, and sulphury [25]. While the system identified the predominant taste characteristics of beer, previous research (i.e., [26]) has suggested that members of the general population (i.e., novices) may not be as sensitive as the electronic tongue, and thus may struggle to identify some of these 
characteristics. In addition, given that past research has found that positive emotions influence perceived 'pleasantness' $[3,16,17]$, and that FaI theory suggests that feelings will influence processing style (i.e., bottom-up or global processing), it was deemed important to include global 'pleasantness' assessments of beer, including the pleasantness of taste and the pleasantness of aftertaste.

The studies outlined above used a variety of stimuli and methods to induce emotion (e.g., static images, sounds, writing or reading emotional stories), although, in the context of beer, only music has been used previously [15]. In the preliminary study presented here, film clips previously found to elicit the matching emotional responses were used [27]. A further departure from Reinoso-Carvalho et al.'s [15] recent work was in the selection of emotions. Reinoso-Carvalho et al. [15] examined perceptual differences with regard to the broad, higher-order dimensions of positive and negative affect (as measured by the Positive and Negative Affect Schedule [PANAS] [28]). Côtè [8] noted that where the focus of evaluation is the broad valence of mood (e.g., positive/negative, pleasant/unpleasant), interpretations are limited and, as such, encouraged the examination of discrete emotional states. The current study therefore focused on two discrete emotional states: one positive (joviality) and the other negative (sadness [29]). The aim of this preliminary study was to assess whether experimentally induced joviality and sadness can influence the perceived taste/flavor of beer. Informed by previous research, and in particular by FaI theory [7], several hypotheses are proposed, which were examined using a combination of null hypothesis significance testing (NHST) and Bayesian analyses.

First, given previous work in related fields (e.g., $[17,19])$, the hypotheses tested with NHSTs are that those induced to experience joviality would rate the beer's sweetness and, by extension, fruitiness as more intense than those induced to experience sadness. It is worth noting at this point that Harrar, Smith, Deroy, and Spence [30] have demonstrated that people sometimes confuse sweetness and fruitiness (see also [31]). In relation to the Bayesian analyses, the alternate hypotheses are that emotions (a model combining joviality and sadness) have an effect on perceived sweetness and fruitiness, whereas the null hypotheses are that emotions have no effect. Second, based on earlier research $[3,16,17]$, and the FaI claim that positive feelings result in increased global processing, it is hypothesized that those induced to experience joviality will rate the beer's pleasantness of taste and pleasantness of aftertaste as being higher than those induced to experience sadness. The hypotheses associated with the Bayesian analyses are that emotions (joviality and sadness) influence the pleasantness of taste and aftertaste, whereas the null hypotheses are that emotions have no effect on such ratings. It is further hypothesized that those induced to feel joviality will rate the beer as being of higher quality and be willing to pay more for it than those induced to experience sadness. The alternate hypotheses associated with the Bayesian analyses are that emotions have an effect on perceived quality and willingness to pay.

\section{Materials and Methods}

\subsection{Participants}

The sample consisted of 32 individuals (17 men, 15 women) from either the general population or the undergraduate population at a regional, Australian university. The participants ranged in age from 18 to 58 years, with a mean of 30.81 years $(S D=11.36)$. The participants had to be at least 18 years of age, as this is the minimum age for legal consumption of alcohol in Australia. The majority of the participants reported that they consumed beer less than once a month $(40.6 \%)$, with the remainder consuming beer either (a) more than once a month but less than once a week $(18.8 \%)$, (b) once a week $(21.9 \%)$, or (c) more than once a week $(18.8 \%)$. The joviality and sadness groups did not differ significantly with respect to their age. However, a $2 \times 2$ chi-square test of independence indicated that there were more men in the joviality group than women, and more women in the sadness group than men $(p=0.034)$. 


\subsection{Materials and Apparatus}

\subsubsection{Emotion Induction Film Clips}

To induce emotion in participants, scenes from two movies were presented. Participants assigned to the 'joviality' condition watched a scene from Wall-E in which two robots dance. This video was three min and $15 \mathrm{~s}$ long. Participants assigned to the 'sadness' condition watched a scene from the Shawshank Redemption where an old man, who has recently been released from prison, hangs himself. This video was six min and $14 \mathrm{~s}$ long. These scenes were selected based on previous research that demonstrated their effectiveness in eliciting the relevant emotional response [27]. Participants viewed the movie scenes on a desktop computer, with a 22-inch monitor. They sat approximately $45 \mathrm{~cm}$ from the screen.

\subsubsection{Beer}

Yenda Pale Ale was given to all participants in all experimental conditions. This beer was $4.5 \%$ alcohol. Each bottle of beer was kept refrigerated at $3{ }^{\circ} \mathrm{C}$ prior to the experiment. Yenda Pale Ale is an Australian beer brewed by the Australian Beer Company [32]. This beer is described as full-bodied and having medium intensity, with a biscuity flavor, and a fruity and floral aroma [32].

\subsubsection{Positive and Negative Affect Schedule-X (PANAS-X [29]).}

The PANAS-X is a 60-item questionnaire used to measure self-reported affective states. The original PANAS [28] was developed to measure the two higher-order dimensions of positive and negative affect. The PANAS- $X$ is an expanded form of the PANAS which, in addition to the higher-order dimensions, includes subscales to measure 11 specific lower-order emotional states reflecting basic negative emotions (fear, sadness, guilt, and hostility), basic positive emotions (joviality, self-assurance, and attentiveness), and other affective states (shyness, fatigue, surprise, and serenity). Participants respond to items (e.g., "cheerful", "distressed") by rating the extent to which they are experiencing each feeling at the present moment on five-point Likert scales, ranging from 1 (very slightly or not at all) to 5 (extremely). Subscale scores are calculated by summing the scores from the relevant items, with higher scores indicating higher levels of the relevant emotion. In this preliminary study, only two subscales were used: joviality and sadness. The joviality subscale was selected as the most appropriate positive emotional scale as, although there is a 'happy' item, there is no happy subscale. The joviality subscale is comprised of the following items: "happy", "joyful", "delighted", "cheerful", "excited", "enthusiastic", "lively", and "energetic". The sadness subscale is comprised of the following items: "sad", "blue", "downhearted", "alone", and "lonely". The joviality and sadness subscales were used as a manipulation check in order to establish that the joviality video did indeed induce joviality, while the sadness video induced sadness. The participants were asked to complete the entire PANAS- $X$ so that they did not guess the specific emotions we were assessing.

The PANAS-X has excellent psychometric properties. The measure exhibits good construct validity [29] and has been shown to have appropriate convergent validity with similar measures (e.g., the Profile of Mood States; [33]). In the initial psychometric testing, Watson and Clark [29] found that the Cronbach's alpha for the joviality subscale was excellent $(\alpha=0.93)$, and good for the sadness subscale $(\alpha=0.86)$. We found similar reliability coefficients for our sample. Specifically, the joviality subscale was excellent $(\alpha=0.94)$, while the sadness subscale was acceptable $(\alpha=0.78)$.

\subsubsection{Beer Taste Perception Questionnaire}

A questionnaire was used to measure taste/flavor perception. This questionnaire consisted of nine items that measured different characteristics of beer: pleasantness of taste, pleasantness of aftertaste, intensity of taste, sweetness, fruitiness of flavor, bitterness, refreshingness, quality, and willingness to pay. These items were selected for a variety of reasons. First, sweetness, bitterness, fruitiness, and intensity of taste have all been established as key sensory attributes of beer [25]. Second, 
pleasantness of taste and aftertaste, quality, and willingness-to-pay are all global valence judgments that have been used in previous research [34], and according to FaI theory, may be influenced by feelings [35]. Lastly, the refreshingness of the beer was included as it is a commonly used descriptor for beer [36], thus making it worthy of further examination.

Participants responded to eight items of this survey using an eleven-point Likert-type scale, which ranged from 0 (Not___ at all) to 10 (Extremely____ . For example, the question "How pleasant would you rate the taste of the beer?" had response options ranging from Not pleasant at all to Extremely pleasant. The 'willingness to pay' item was answered by providing a monetary amount (in Australian dollars) of how much each participant was willing to pay for a full glass of the beer they consumed in the experiment. Items in this questionnaire were randomized in an attempt to control for order effects.

\subsubsection{Demographic Questionnaire}

To gather demographic and other important information, a post-experiment questionnaire was used. This questionnaire had the participants provide their age, gender, and drinking habits (e.g., how often they consume beer). The questions relating to drinking habits were answered on four-point response scales ranging from less than once a month to more than once a week.

\subsection{Procedure}

The study proceeded following approval from Federation University's Human Research Ethics Committee (Reference number: A18-086). Participants were recruited via social media, snowballing, and advertisements on noticeboards around a regional university campus. The advertising material did not fully disclose the aim of the experiment, and stated that the purpose of the experiment was to investigate the relationship between movies and beer flavor perception. According to the FaI theory, feelings are just like any other form of information in that individuals may discount them if they become aware that they are the product of an unrelated source. That is, if the individual questions whether their emotional state (e.g., joviality induced by visual stimuli) is related to the situation or object currently being evaluated (e.g., judging the flavor of a beer), there can be a discounting effect such that the influence of emotion on that evaluation disappears [7]. Alternatively, there may also be an augmentation effect such that there is an adjustment in the opposite direction. Therefore, in order to investigate the influence of feelings on flavor perception, we withheld information so that participants did not question the manipulation of their emotions.

Each participant was tested individually. Prior to participation, each participant was supplied with a statement outlining ethical information with regard to voluntary participation, the right to withdraw at any time, and anonymity of the information they provide. Some participants were first year psychology students at the regional university, and they received course credit for their participation. No other rewards were offered. After reading the statement, if participants agreed to take part they signed a consent form.

The participants were allocated to either the joviality or sadness group using a pre-determined randomization method. Sixteen participants were allocated to each group. Participants were then given a brief overview of what the experiment would entail, and roughly how long each stage would take. Following this overview, participants watched their allocated film clip in order to induce either joviality or sadness. The experiment was conducted in a small room with controlled artificial lighting, as it has been shown that variability in lighting can influence mood [37]. Whilst participants were engaged in the movie-watching task, the experimenter poured $250 \mathrm{~mL}$ of beer into a clear plastic cup, ensuring that the beer's head was similar for each participant. This was done out-of-sight of the participants. After watching the video, participants were asked to sample the beer and complete a hardcopy of the Beer Taste Perception Questionnaire. The participants were encouraged to take small sips of the beer as they evaluated its characteristics. They were informed that they could consume as much of the beer as they liked. Following this, any unconsumed beer and the plastic cups were removed from the room and disposed of. Participants were then asked to rate their current affective 
state using the PANAS-X and fill in the Demographics Questionnaire. All participants completed the experiment in this order to (a) minimize the time between emotion induction and beer tasting, (b) negate demand characteristics that might be present had we presented the PANAS-X before beer tasting, and (c) avoid making individuals aware of their induced emotional state by completing the PANAS-X, such that they might discount it when evaluating beer [7]. After completing the experiment, the participants were debriefed and made aware of the aim of the study, and the hypothesis of the study was revealed. The experiment took approximately $25 \mathrm{~min}$ to complete.

\subsection{Statistical Analyses}

Given the small sample size, in addition to the standard (frequentist) independent-measures $t$-tests, we present effect sizes, 95\% Confidence Intervals (CIs), and performed Bayesian independent-measures $t$-tests. Bayesian analyses are being used with increased regularity in psychological research (see [38]). Here, the Bayesian $t$-test determines the Bayes Factor comparing the fit of the data under the null hypothesis (i.e., that the effect is zero) and the alternative hypothesis. The model with the largest Bayes Factor is considered to be the best-fitting model, as it has the greatest evidence and best explains the data. Frequentist metrics such as $p$-values, as used in Fisherian NHSTs, are not only biased against the null hypothesis [39], but also only enable the evaluation of evidence for the alternative hypothesis with no quantification of the level of evidence for the null. In other words, a $p$-value $>0.05$ fails to distinguish between a lack of evidence for either the null or alternate hypotheses, and does not indicate the level of evidence for the null hypothesis. In this kind of inferential setting, Bayes Factors can directly quantify the amount of evidence for one hypothesis (or model) relative to another hypothesis (or model), often a null model. A Bayes Factor of $\mathrm{BF}_{10}=10$, for example, would indicate that, given the data, $\mathrm{H}_{1}$ (the alternate) is ten times more likely than the null. Based on Jeffreys [40], Andraszewicz et al. [39] developed a series of labels for Bayes Factors giving some indication of what could be considered reasonable evidence (e.g., $\mathrm{BF}_{10}=1$ is anecdotal, $\mathrm{BF}_{10}=3$ is moderate, and a $\mathrm{BF}_{10}$ $=100$ is extreme evidence). Bayesian statistics were calculated using Jeffreys's Amazing Statistics Program (JASP version 0.9.0.1) [41].

The Bayes Factors used here are presented in standard form of $\mathrm{BF}_{10}=\mathrm{X}$ where the subscript 10 indicates that the Bayes Factor is relative to the null model (evidence for the alternate). The $\mathrm{X}$ is the Bayes Factor itself.

\section{Results}

\subsection{Preliminary Analyses}

\subsubsection{Missing Values Analysis}

Missing values were present in the dataset due to non-response. As such, a missing values analysis and Little's Missing Completely at Random (MCAR) test were conducted. The MCAR test on data missing from the PANAS-X showed that there was no pattern to the missing data $\left(\chi^{2}=22.29 ; d f=31\right.$; $p=0.87$ ), indicating responses were missing completely at random. That said, the missing values analysis indicated that more than $5 \%$ of responses were missing from several subscales, including sadness $(6.3 \%)$ and joviality (6.3\%). Field [42] suggests that missing data exceeding $5 \%$ may be cause for concern. Although the missing data will not bias the findings of the study, they will reduce statistical power due to the reduced sample size. Thus, multiple imputation was performed with 54 data-points in total being imputed (i.e., estimates based on responses to other items are substituted for missing values), including 10 for the joviality subscale, and eight for the sadness subscale.

\subsubsection{Assumption Testing}

The data were assessed to ensure the assumptions of normality and homogeneity of variance were met. The pleasant taste, pleasant aftertaste, and intensity of taste data violated the assumption 
of normality. These variables were negatively skewed. As such, a reflection of the data was carried out, and then the data were log-transformed. This transformation successfully normalized the data. We then carried out the NHSTs with transformed data and with untransformed data. The outcomes from these analyses were, for all intents and purposes, identical and, as such, we will report the analyses using untransformed data. Here, the interested reader is referred to the literature suggesting that certain parametric tests are robust against minor violations of normality [43]. Finally, homogeneity of variance was assessed via Levene's Test of Equality of Error Variances, with some variables violating this assumption. When this occurred, we used figures associated with 'Equal variances not assumed'.

\subsection{Descriptive Statistics}

Participants in both groups rated the beer on eight different characteristics of taste/flavor and willingness to pay. Means and standard deviations for each of the variables are reported in Table 1 for the joviality and sadness groups.

Table 1. Descriptive statistics for beer flavor characteristics for joviality and sadness groups.

\begin{tabular}{ccccc}
\hline & \multicolumn{2}{c}{ Joviality } & \multicolumn{2}{c}{ Sadness } \\
\hline Variables & $\boldsymbol{M}$ & $\boldsymbol{S D}$ & $\boldsymbol{M}$ & $\boldsymbol{S D}$ \\
\hline Pleasant taste & 7.56 & 2.10 & 5.19 & 2.83 \\
\hline Pleasant aftertaste & 6.06 & 2.54 & 4.38 & 3.18 \\
\hline Sweetness & 5.06 & 2.32 & 2.94 & 2.54 \\
\hline Bitterness & 5.06 & 2.46 & 6.88 & 2.50 \\
\hline Fruitiness & 3.81 & 2.83 & 3.50 & 2.68 \\
\hline Refreshingness & 6.81 & 2.32 & 5.88 & 2.63 \\
\hline Intense taste & 7.00 & 1.32 & 6.50 & 2.19 \\
\hline Quality & 7.50 & 0.97 & 5.94 & 2.14 \\
\hline Willingness to pay (AUD) & $\$ 6.28$ & $\$ 1.32$ & $\$ 4.94$ & $\$ 1.29$ \\
\hline Note: $M=$ Mean, $S D=$ Standard Deviation.
\end{tabular}

\subsection{Manipulation Checks}

Two independent-samples $t$-tests were conducted to assess whether the joviality and sadness conditions caused people to differ on the relevant subscales of the PANAS-X. This was done as a manipulation check in order to ascertain whether the film clips induced feelings of joviality or sadness in participants, as expected. A Bonferroni-corrected, independent-samples $t$-test on subjective ratings of joviality was conducted and revealed that the joviality group $(M=28.25, S D=6.69)$ scored significantly higher than did the sadness group $(M=19.71, S D=8.98), t(28)=2.97, p=0.003$, two-tailed, $d=1.08$ (95\% CI: 2.73-13.37). A second Bonferroni-corrected, independent-samples $t$-test found a statistically significant difference in sadness scores for the two groups. Participants in the induced joviality group $(M=6.13, S D=1.96)$ were less sad than those in the induced sadness group $(M=8.71, S D=3.05)$, $t(28)=2.97, p=0.007$, two tailed, $d=1.01$ (95\% CI: $-4.11--0.65)$. As such, preliminary evidence shows that the film clips appear not only to have induced the relevant emotion, but that this emotional state persisted until after the beer ratings were completed. It is worth noting that, after correcting for multiple comparisons, the joviality and sadness conditions did not cause people to differ on any other subscales of the PANAS-X. Note that this validates the selection of the video stimuli and PANAS-X subscales, particularly joviality, as being appropriate.

\subsection{NHST Independent-Samples t-Tests}

Following this, frequentist independent-samples $t$-tests were used to investigate the hypotheses. These tests can be seen in Table 2 . 
Table 2. Independent-samples $t$-tests for beer flavor characteristics assessing differences between the joviality and sadness groups. We subjected our analyses to Holm-Bonferroni corrections and, as there were nine tests, we applied a stringent critical $p$-value of 0.006 (i.e., $0.05 / 9$ ).

\begin{tabular}{cccccc}
\hline DVs. & $d f$ & $t$ & $p$ & $d$ & 95\% CI \\
\hline Pleasant taste & $27.64 *$ & 2.70 & 0.012 & 0.95 & $0.58-4.18$ \\
Pleasant aftertaste & $28.61^{*}$ & 1.66 & 0.108 & 0.58 & $-0.40-3.77$ \\
Sweetness & 30 & 2.47 & 0.019 & 0.87 & $0.37-3.88$ \\
Bitterness & 30 & -2.07 & 0.048 & 0.73 & $-3.60--0.02$ \\
Fruitiness & 30 & 0.32 & 0.751 & 0.11 & $-1.68-2.31$ \\
Refreshingness & 30 & 1.07 & 0.293 & 0.38 & $-0.85-2.73$ \\
Intense taste & 30 & 0.78 & 0.440 & 0.28 & $-0.81-1.81$ \\
Quality & $20.85 *$ & 2.66 & 0.015 & 0.94 & $0.34-2.79$ \\
Willingness to pay (AUD) & 30 & 2.92 & 0.007 & 1.03 & $0.40-2.29$ \\
\hline
\end{tabular}

Notes: DVs. $=$ Dependent Variables, ${ }^{*}=$ The assumption of equal variances was violated.

Conventionally, there were statistically significant differences between the emotion conditions in pleasantness of taste, sweetness, bitterness, quality, and willingness to pay. However, after the Holm-Bonferroni correction (see Table 1), none of the analyses achieved statistical significance, although willingness to pay came very close. However, some of the results produced medium-to-large effect sizes. As a measure of effect size, Cohen's $d$ statistics were calculated to determine the magnitude of the difference between the groups. The magnitude of the difference was classified as either small $(0.2)$, medium (0.5), or large (0.8) based on Cohen's guidelines [44,45]. Pleasant taste, sweetness, quality, and willingness to pay all had large effect sizes, as well as $95 \%$ CIs that did not cross zero. It is important to note that one result (i.e., $p$-value) suggests that the observed differences were due only to sampling or measurement error, while the other (e.g., effect size) suggests that there is an effect of emotion on perception. Further Bayesian analyses were conducted which were able to provide support for one of these positions.

\subsection{Bayesian Independent-Samples t-Tests}

Bayesian independent-samples $t$-tests were run on the subjective ratings of the beer's properties (see Table 3).

Table 3. Bayes factor independent-samples $t$-tests for beer flavor characteristics assessing evidence for the null and alternative hypotheses.

\begin{tabular}{ccc}
\hline DVs. & $\boldsymbol{B F}_{\mathbf{1 0}}$ & Support for $\mathbf{H}_{\mathbf{1}}$ \\
\hline Pleasant taste & 4.58 & Moderate \\
Pleasant aftertaste & 0.94 & Anecdotal \\
Sweetness & 3.08 & Moderate \\
Bitterness & 1.63 & Anecdotal \\
Fruitiness & 0.35 & Anecdotal \\
Refreshingness & 0.52 & Anecdotal \\
Intense taste & 0.43 & Anecdotal \\
Quality & 4.29 & Moderate \\
Willingness-to-pay (AUD) & 6.93 & Moderate \\
\hline \multicolumn{2}{c}{ Note: BF = Bayes Factor. }
\end{tabular}

An example interpretation of the Bayesian analyses can be provided through comparison to the frequentist independent-samples $t$-test assessing 'willingness to pay'. The latter analysis shows that people induced to experience joviality tended to be willing to pay more for the beer $(M=\$ 6.28$, $S D=\$ 1.32)$ than those participants induced to experience sadness instead $(M=\$ 4.94, S D=\$ 1.29)$, $t(30)=2.92, p=0.007, d=1.03$. In contrast, the Bayesian analysis examined the data by estimating a Bayes Factor and comparing the fit of the data under the null hypothesis and alternative hypothesis. 
An estimated Bayes Factor suggested that the data were 6.93:1 in favor of the alternative hypothesis. That is, the data were 6.93 times more likely to occur under the model including an effect for emotion (i.e., alternative hypothesis), rather than the model without it (i.e., the null hypothesis). According to Andraszewicz et al. [39], this would be interpreted as moderate evidence.

\section{Discussion}

The aim of the current preliminary study was to investigate whether or not emotion influenced the perception of different characteristics of beer. On the basis of previous research [46], the hypotheses related to the NHSTs were that those participants induced to feel joviality would report the beer as tasting sweeter and fruitier, relative to those participants induced to feel sadness. In relation to the Bayesian analyses, it was hypothesized that emotions (a model combining joviality and sadness) would have an effect on perceived sweetness and fruitiness. Based on prior research [3,16], and FaI theory, the participants induced to experience joviality (compared to sadness) were expected to rate the beer as having a more pleasant taste, and aftertaste. The Bayesian hypotheses were that emotions would have an effect on pleasantness of taste and aftertaste. It was also hypothesized that participants induced to experience joviality would rate the beer as being of higher quality and would be willing to pay more for it, relative to participants experiencing sadness. The Bayesian hypotheses were that emotions would have an effect on perceived quality and willingness to pay.

Although the NHST with adjusted critical values failed to support these hypotheses, the results of the Bayesian analyses indicated that there was moderate evidence to suggest that some of the alternative hypotheses were supported, suggesting that induced emotions influenced people's ratings of the pleasantness of taste, sweetness, quality, and the amount they were willing to pay for the beer. These findings are supported by the large effect sizes and 95\% CIs for pleasantness of taste, sweetness, quality, and willingness to pay in the NHSTs. There has been commentary in the literature suggesting that effect size should be considered a more salient piece of information relative to $p$-values [47]. The effect size signifies the magnitude of the difference between two groups [48], regardless of sample size [49]. Given the large effect sizes, and the findings from the Bayesian analyses, we have preliminary evidence that there is an effect of emotion, specifically joviality and sadness, on the perception of certain characteristics of beer, and will interpret the findings accordingly.

\subsection{Flavor}

Six taste/flavor variables were measured (i.e., pleasantness of taste, pleasantness of aftertaste, sweetness, bitterness, fruitiness, and intensity). Although the NHSTs were non-significant, the results revealed that pleasantness of taste and sweetness produced large effect sizes, and 'moderate' evidence for the alternative hypothesis in the Bayesian analyses. The latter findings are consistent with the FaI theory, according to which feelings can provide a source of information that informs perception [7] and be unconsciously attributed to an object the person is evaluating [24]. Feelings of joviality induced by the Wall-E video, and feelings of sadness induced by the Shawshank Redemption video, appear to have been unconsciously (mis)attributed to the beer, and thus we have preliminary evidence showing that each participant's emotion influenced their taste perception.

Although we have preliminary evidence suggesting that emotions induced by video content influenced the multisensory flavor experience of beer, an interesting question is whether it was joviality that increased beer pleasantness ratings, or if it was induced sadness that lowered ratings of pleasantness of taste. A previous study using the same brand of beer and the same 11-point rating scale used here, but with no emotional manipulation, found that the mean 'pleasantness of taste' rating was approximately six (6) [34]. Given this information, the results of the current study (see Table 1) suggest that induced joviality $(M=7.56, S D=2.10)$ increased the 'pleasantness of taste' rating, while induced sadness seems to have decreased it $(M=5.19, S D=2.83)$. In a numerical assessment, induced joviality appears to have had a larger effect than induced sadness. Similarly, Mirabito et al.'s [34] mean sweetness rating was approximately four. As such, and given the results in Table 1, induced joviality 
$(M=5.06, S D=2.32)$ seems to have increased the perceived sweetness of the beer, while induced sadness $(M=2.94, S D=2.54)$ seems to have decreased it. The results are also consistent with those from previous research $[3,16,17,19]$ in that people induced to feel joviality rated the beer as tasting sweeter and more pleasant than those induced to feel sadness.

The effect sizes and confidence intervals seem to be consistent with differences in processing styles as suggested by FaI theory. 'Pleasantness' is arguably a global assessment in that it does not focus on specifics. According to FaI, positive feelings foster a processing style in which assessments are made less analytically $[7,8,23]$. Accordingly, participants in the joviality group may have been more inclined to make a global assessment of the beer, which led to higher ratings of the overall pleasantness of the taste of the beer. Negative feelings (e.g., sadness) are argued to foster analytic processing, with individuals attempting, either consciously or unconsciously, to identify the source of their current problematic situation, in order to change their mood to one that is more positive [7]. In order to do so, the individual must first conduct a comprehensive analysis of the current situation [50]. As such, greater scrutiny is applied to specific details of the beer, with the person attempting to identify factors that are contributing to their current situation. Consequently, individuals may rate as higher any factors that are potentially contributing to their mood.

Some of the results reported here provide support for the different processing styles as suggested by FaI theory. Specifically, the means for bitterness and sweetness in the joviality group were identical (5.06; see Table 1), whilst the sadness group resulted in more extreme and divergent ratings (sweetness $=2.94$; bitterness $=6.88$ ). This suggests that people induced to experience sadness may have been more discerning and apply greater effort to processing of the taste/flavor. We put forward this interpretation tentatively because we have not tested processing style, and a fruitful area of future work might be to do this directly.

Another fruitful area for future research might be to target regular beer drinkers. In our study, many of the participants reported drinking beer less than once a month and although our goal was to extrapolate the preliminary findings from our sample to the general population, screening for regular beer drinkers would provide results that pertain specifically to beer drinkers. Here, the prohibitive sample size limited the ability to analyze specific groups. On this point, university students comprised a significant proportion of our sample. Future research should endeavor to recruit a sample that more closely represents either beer drinkers or the general population.

\subsection{Individual Differences}

The perceived quality of the beer and how much each participant was willing to pay for the beer both achieved large effect sizes, and 'moderate' evidence for the alternative according to the Bayesian analyses but, again, failed to achieve statistical significance in the NHSTs. Here, participants in the joviality group rated the beer as being of higher quality than did those in the sadness group; they were also willing to pay more for it than were those people in the latter group. The results of the present study suggest that induced emotion may have provided a source of (unconscious) information when participants evaluated the beer (see [7,24,51] for similar arguments). In terms of quality, we have preliminary evidence suggesting that a feeling of joviality is (unknowingly) used as a source of information suggesting that the beer is 'good', and thus the ratings of the beer's quality were higher than those who were induced to experience sadness. Taken together, this preliminary evidence appears to support the FaI theory [7] in that positive feelings promote a global processing style [8,23], and perceived quality arguably constitutes a global assessment of beer.

The same explanation can be applied to willingness to pay. That is, it is obviously unlikely that individuals would pay more for a beer that they believed was bad or unpleasant. As the beers were the same, and the only difference was in terms of the emotion induced, it can be tentatively assumed that people's experience of joviality (or sadness) was used to infer that the beer was good (or bad). Again, however, it is difficult to determine whether a positive emotion increased the amount people were willing to pay, or if a negative emotion decreased the amount. If we use Mirabito et al.'s [34] work to 
establish a baseline, they found that the average amount people were willing to pay was $\sim \$ 5.30$ AUS. In the jovial condition here, the average was $\$ 6.28$, while in the sad condition it was $\$ 4.94$. As such, it appears as though inducing people to experience joviality will increase the amount they are willing to pay, more than inducing sadness will decrease this amount. That said, and although we had a comparison group, future research could include a control condition (e.g., an indifferent affective state).

\section{Conclusions}

This preliminary research has real-world applications. Research (e.g., [15]) shows that music can influence emotions which, in turn, can influence the multisensory perception of beer. Here, we show that other elements of the environment (e.g., what is played on television sets in establishments that serve beer) may also influence the drinking experience. However, it is important to stress that this conclusion depends on whether effect sizes, CIs, and Bayesian analyses or $p$-values are used to interpret the results. If establishments that serve beer (e.g., restaurant, bars) are able to manipulate the environment such that their patrons are induced to experience joviality, there should be a tendency to rate the beers they serve as being sweeter, of better quality, and having a more pleasant taste, relative to those establishments where patrons experience sadness. Importantly, they may also be willing to pay more. Conversely, if the establishment serves poor-quality beer, sadder people will be more likely to critically analyze it! From a theoretical perspective, this preliminary study is the first to isolate the specific emotions of joviality and sadness as potentially influencing the perception of beer.

A limitation of this study is the small sample size which, depending on the analyses one favors, limits the generalizability of the results and conclusions that can be drawn. That said, this area of research is relatively new and some of the existing studies, upon which this study was built, have similarly small sample sizes (e.g., $n=48$ [3]; $n=49$ [19]). Clearly, additional research with larger samples that more fully represent the population is needed. Nevertheless, this preliminary study contributes to our understanding of the role of emotions in beer perception, from the broader higher-order dimensions of positive and negative affect to specific basic emotions, and at the very least, provides a fascinating direction for future research.

Author Contributions: Conceptualization, B.D., S.W., and G.V.D.; methodology, B.D., S.W., and G.V.D.; formal analysis, B.D. and G.V.D.; investigation, B.D.; writing—original draft preparation, B.D.; writing-review and editing, G.V., S.W., J.T., and C.S.; supervision, S.W. and G.V.D.; project administration, B.D. All authors have read and agreed to the published version of the manuscript.

Funding: This research was funded by the Arts and Humanities Research Council (AHRC), grant number AH/L007053/1 awarded to C.S.

Conflicts of Interest: The authors declare no conflict of interest. The funders had no role in the design of the study; in the collection, analyses, or interpretation of data; in the writing of the manuscript, or in the decision to publish the results.

\section{References}

1. Brosch, T.; Scherer, K.R.; Grandjean, D.; Sander, D. The impact of emotion on perception, attention, memory, and decision-making. Swiss Med. Wkly. 2013, 143, w13786. [CrossRef] [PubMed]

2. Riener, C.R.; Stefanucci, J.K.; Proffitt, D.R.; Clore, G. An effect of mood on the perception of geographical slant. Cogn. Emot. 2011, 25, 174-182. [CrossRef] [PubMed]

3. Macht, M.; Roth, S.; Ellgring, H. Chocolate eating in healthy men during experimentally induced sadness and joy. Appetite 2002, 39, 147-158. [CrossRef] [PubMed]

4. Ekkekakis, P. Affect, mood, and emotion. In Measurement in Sport and Exercise Psychology; Tenenbaum, G., Eklund, R.C., Kamata, A., Eds.; Human Kinetics: Champaign, IL, USA, 2012; pp. 321-332.

5. Ekkekakis, P. The Measurement of Affect, Mood, and Emotion: A Guide for Health-Behavioral Research; Cambridge University Press: New York, NY, USA, 2013.

6. Russell, J.A.; Barrett, L.F. Core affect, prototypical emotional episodes, and other things called emotion: Dissecting the elephant. J. Personal. Soc. Psychol. 1999, 76, 805-819. [CrossRef] 
7. Schwarz, N. Feelings-as-information theory. In Handbook of Theories of Social Psychology; Van Lange, P., Kruglanski, A., Higgins, E.T., Eds.; Sage: Newbury Park, CA, USA, 2012; pp. 289-308.

8. Côtè, $\mathrm{S}$. Reconciling the feelings-as-information and hedonic contingency models of how mood influences systematic information processing. J. Appl. Soc. Psychol. 2005, 35, 1656-1679. [CrossRef]

9. Vanderhaegen, B.; Neven, H.; Coghe, S.; Verstrepen, K.J.; Derdelinckx, G.; Verachtert, H. Bioflavoring and beer refermentation. Appl. Microbiol. Biotechnol. 2003, 62, 140-150. [CrossRef]

10. Verbelen, P.J.; De Schutter, D.P.; Delvaux, F.; Verstrepen, K.J.; Delvaux, F.R. Immobilized yeast cell systems for continuous fermentation applications. Biotechnol. Lett. 2006, 28, 1515-1525. [CrossRef]

11. Smith, G.D. Beer: A Global History; Reaktion Books: London, UK, 2014.

12. Oliver, G. The Oxford Companion to Beer; Oxford University Press: Oxford, UK, 2011.

13. IWSR Forecast: Global Alcohol Consumption to Hit 28bn Cases and \$1.07tn by 2022. Available online: https://www.theiwsr.com/wp-content/uploads/IWSR-Press-Release_IWSR-Forecast-Global-alcoholconsumption-to-hit-28bn-cases-and-USD1.07tn-by-2022_7Aug2018.pdf (accessed on 8 August 2019).

14. Kirin Beer University Report Global Beer Consumption by Country in 2017. Available online: https: //www.kirinholdings.co.jp/english/news/2018/1220_01.html (accessed on 11 September 2019).

15. Reinoso-Carvalho, F.; Dakduk, S.; Wagemans, J.; Spence, C. Not just another pint! The role of emotion induced by music on the consumer's tasting experience. Multisens. Res. 2019, 32, 367-400. [CrossRef]

16. Willner, P.; Healy, S. Decreased hedonic responsiveness during a brief depressive mood swing. J. Affect. Disord. 1994, 32, 13-20. [CrossRef]

17. Noel, C.; Dando, R. The effect of emotional state on taste perception. Appetite 2015, 95, 89-95. [CrossRef]

18. Plassmann, H.; O'Doherty, J.; Shiv, B.; Rangel, A. Marketing actions can modulate neural representations of experienced pleasantness. Proc. Natl. Acad. Sci. USA 2008, 105, 1050-1054. [CrossRef] [PubMed]

19. Wang, Q.; Spence, C. "A sweet smile": The modulatory role of emotion in how extrinsic factors influence taste evaluation. Cogn. Emot. 2018, 32, 1052-1061. [CrossRef] [PubMed]

20. Västfjäll, D. Emotion induction through music: A review of the musical mood induction procedure. Musicae Sci. 2001, 5, 173-211. [CrossRef]

21. Schwarz, N.; Clore, G.L. Mood, misattribution, and judgments of well-being: Informative and directive functions of affective states. J. Personal. Soc. Psychol. 1983, 45, 513-523. [CrossRef]

22. Brackett, M.A.; Floman, J.L.; Ashton-James, C.; Cherkasskiy, L.; Salovey, P. The influence of teacher emotion on grading practices: A preliminary look at the evaluation of student writing. Teach. Teach. 2013, 19, 634-646. [CrossRef]

23. Hunsinger, M.; Isbell, L.M.; Clore, G.L. Sometimes happy people focus on the trees and sad people focus on the forest: Context-dependent effects of mood in impression formation. Personal. Soc. Psychol. Bull. 2012, 38, 220-232. [CrossRef]

24. Pham, M.T. The logic of feeling. J. Consum. Psychol. 2004, 14, 360-369. [CrossRef]

25. Rudnitskaya, A.; Polshin, E.; Kirsanov, D.; Lammertyn, J.; Nicolai, B.; Saison, D.; Delvaux, F.R.; Legin, A. Instrumental measurement of beer taste attributes using an electronic tongue. Anal. Chim. Acta 2009, 646, 111-118. [CrossRef]

26. Peron, R.M.; Allen, G.L. Attempts to train novices for beer flavor discrimination: A matter of taste. J. Gen. Psychol. 1988, 115, 403-418. [CrossRef]

27. Gabert-Quillen, C.; Bartolini, E.; Abravanel, B.; Sanislow, C. Ratings for emotion film clips. Behav. Res. Methods 2015, 47, 773-787. [CrossRef]

28. Watson, D.; Clark, L.A.; Tellegen, A. Development and validation of brief measures of positive and negative affect: The PANAS scales. J. Personal. Soc. Psychol. 1988, 54, 1063-1070. [CrossRef]

29. Watson, D.; Clark, L.A. The PANAS-X: Manual for the Positive and Negative Affect Schedule-Expanded Form; University of Florida: Gainesville, FL, USA, 1994.

30. Harrar, V.; Smith, B.; Deroy, O.; Spence, C. Grape expectations: How the proportion of white grape in Champagne affects the ratings of experts and social drinkers in a blind tasting. Flavour 2013, 2, 25. [CrossRef]

31. Spence, C.; Velasco, C.; Knoeferle, K. A large sample study on the influence of the multisensory environment on the wine drinking experience. Flavour 2014, 3, 8. [CrossRef]

32. The Yenda Range. Available online: https://www.yendabeer.com.au/our-brews/ (accessed on 11 September 2019).

33. McNair, D.M.; Lorr, M.; Droppleman, L.F. Manual: Profile of Mood States; Educational and Industrial Testing Service: San Diego, CA, USA, 1974. 
34. Mirabito, A.; Oliphant, M.; Van Doorn, G.; Watson, S.; Spence, C. Glass shape influences the flavour of beer. Food Qual. Prefer. 2017, 62, 257-261. [CrossRef]

35. Schwarz, N. Feelings as information: Implications for affective influences on information processing. In Theories of Mood and Cognition: A User's Guidebook; Martin, L.L., Clore, G.L., Eds.; Taylor \& Francis Group: New York, NY, USA, 2013; pp. 159-176.

36. Guinard, J.-X.; Souchard, A.; Picot, M.; Rogeaux, M.; Sieffermann, J.-M. Determinants of the thirst-quenching character of beer. Appetite 1998, 31, 101-115. [CrossRef]

37. Küller, R.; Ballal, S.; Laike, T.; Mikellides, B.; Tonello, G. The impact of light and colour on psychological mood: A cross-cultural study of indoor work environments. Ergonomics 2006, 49, 1496-1507. [CrossRef]

38. Rouder, J.N.; Haaf, J.M.; Vandekerckhove, J. Bayesian inference for psychology, Part IV: Parameter estimation and Bayes factors. Psychon. Bull. Rev. 2018, 25, 102-113. [CrossRef]

39. Andraszewicz, S.; Scheibehenne, B.; Rieskamp, J.; Grasman, R.P.; Verhagen, A.J.; Wagenmakers, E.-J. An introduction to Bayesian hypothesis testing for management research. J. Manag. 2015, 41, 521-543. [CrossRef]

40. Jeffreys, H. The Theory of Probability, 3rd ed.; Oxford University Press: Oxford, UK, 1961.

41. JASP; Version 0.9.0.1; JASP Team, 2018.

42. Field, A. Discovering Statistics Using SPSS, 2nd ed.; Sage: London, UK, 2005.

43. Tabachnick, B.G.; Fidell, L.S. Using Multivariate Statistics, 6th ed.; Pearson Education: Boston, MA, USA, 2013.

44. Cohen, J. Statistical Power Analysis for The Behavioral Sciences, 2nd ed.; Lawrence Erlbaum Associates: Mahwah, NJ, USA, 1988.

45. Morris, P.E.; Fritz, C.O. Effect sizes in memory research. Memory 2013, 21, 832-842. [CrossRef]

46. Pollatos, O.; Kopietz, R.; Linn, J.; Albrecht, J.; Sakar, V.; Anzinger, A.; Schandry, R.; Wiesmann, M. Emotional stimulation alters olfactory sensitivity and odor judgment. Chem. Senses 2007, 32, 583-589. [CrossRef]

47. Wilkinson, L. Statistical methods in psychology journals. Am. Psychol. 1999, 54, 594-604. [CrossRef]

48. Sullivan, G.M.; Feinn, R. Using effect size-Or why the p value is not enough. J. Grad. Med. Educ. 2012, 4, 279. [CrossRef]

49. Sun, S.; Pan, W.; Wang, L.L. A comprehensive review of effect size reporting and interpreting practices in academic journals in education and psychology. J. Educ. Psychol. 2010, 102, 989-1004. [CrossRef]

50. Isen, A.M. An influence of positive affect on decision making in complex situations: Theoretical issues with practical implications. J. Consum. Psychol. 2001, 11, 75-85. [CrossRef]

51. Clore, G.L.; Gasper, K.; Garvin, E. Affect as information. In Handbook of Affect and Social Cognition; Forgas, J.P., Ed.; Lawrence Erlbaum Associates: Mahwah, NJ, USA, 2001; pp. 121-144. 